CERN-PPE/91-192

22 October 1991

\title{
Ramsey Technique Applied in a Penning Trap Mass Spectrometer
}

\author{
G. Bollen, H.-J. Kluge, T. Otto, G. Savard and H. Stolzenberg, \\ Institut für Physik, Universität Mainz, \\ D-6500 Mainz, Fed. Rep. Germany
}

and the ISOLDE Collaboration, PPE Div., CERN, Geneva, Switzerland

\begin{abstract}
The Ramsey method has been applied in an experiment aiming for accurate mass determination of unstable isotopes. The ion motion in a Penning trap has been excited with time-separated oscillatory fields and Ramsey fringes were observed in the case of dipole and quadrupole excitation. The experimental resonances are in good agreement with theory. Further applications of the technique are discussed.
\end{abstract}

(IS130)

Submitted to Nucl. Instrum. \& Methods B 



\section{INTRODUCTION}

In 1949 Norman F. Ramsey invented the technique of separated oscillatory fields in a molecular beam experiment [1]. Since this date his idea of first preparing a physical system in a well defined state, then letting it evolve in time or space and finally to interrogate its state again has found a large number of applications [2]. The cesium clock used as time standard is presently the most important one. Experiments aiming for highest accuracy are making use of this technique; the lowest limit for the electric dipole moment of the neutron was determined in an experiment using spin polarization as preparing and analyzing step. Between these steps the neutron spins were allowed to precess in a weak magnetic and strong electric field for an extended period of time [3]. High-accuracy atomic physics or metrological experiments use laser light to excite atoms either space or time separated. The time-separated excitation together with the storage of the particles in space for nearly infinite periods of time by means of ion traps is certainly one of the most promising ways towards a new time standard. Beautiful experiments have been performed, one of the most recent being a test of the linearity of quantum mechanics by searching for a change in the transition frequency between two states in a Be ion while undergoing this transition [4].

Precision mass experiments have only applied the Ramsey technique in cases where the mass determination is performed via a frequency measurement. The cyclotron frequency of ions in a strong magnetic field is determined by a Ramsey-like technique in Smith's RF-mass spectrometer [5]. Presently such a spectrometer is set up at CERN for the determination of the mass ratio of proton to antiproton $m(p) / m(\bar{p})[6]$. The ratio of $m(C O) / m\left(N_{2}\right)$ was recently determined with an accuracy of $<10^{-9}$ by a variant of Ramsey's method in a Penning trap experiment [7].

In this paper, we report on the application of the Ramsey method in an experiment aiming for the mass determination of heavy radioactive isotopes. The mass of these ions is determined with accuracies of $10^{-7}$ by means of a Penning trap mass spectrometer with a single RF-excitation [8]. Ramsey fringes have been observed by time-separated excitation of the motion of stored stable ions. This technique can help to increase the accuracy in mass determination.

\section{THEORETICAL CONSIDERATIONS}

The Penning trap allows the confinement of charged particles in a magnetic field $B$ for an extended period and the observation or excitation of their motions. The confine- 
ment is achieved by an electrode configuration creating an axially symmetric electrostatic quadrupole field which prevents the ions from escaping along the magnetic field lines. In general this configuration consists of three electrodes; two endcaps and one ring electrode which are hyperboloids of revolution. Due to the additional electric force the motion of an ion with mass $m$ and charge $q$ is no longer a pure cyclotron motion with frequency

$$
\nu_{c}=\frac{1}{2 \pi} \frac{q}{m} \cdot B
$$

but a superposition of three harmonic eigenmotions; a slow drift around the trap axis called magnetron motion with frequency $\nu_{-}$, a modified cyclotron motion with frequency $\nu_{+}$and an axial oscillation with frequency $\nu_{z}[9]$. These harmonic motions can be driven by oscillating electric dipole fields. If the amplitude of the motion is initially zero it will increase linearly in time if the RF-field has the same frequency as the particular eigenmotion.

There exists a relation between the radial eigenfrequencies which is important for all high-accuracy mass experiments; the sum of the radial eigenfrequencies equals the cyclotron frequency of an ion in a pure magnetic field:

$$
\nu_{+}+\nu_{-}=\nu_{c}
$$

This allows a mass determination without the knowledge of the electric trapping field. Furthermore the ion motion can be directly excited at this sum frequency with an azimuthal quadrupole field. Such an excitation couples magnetron and cyclotron motion leading to a periodic conversion from one motion into the other [10].

In general the excitation of the ion motion changes its energy. The excitation of the cyclotron motion by a dipole field with frequency $\nu_{+}$increases the kinetic energy of the motion quadratically in time. In the case of a quadrupole excitation at $\nu_{+}+\nu_{-}$, the conversion of magnetron into cyclotron motion results as well in a change in energy since in general $\nu_{+} \gg \nu_{-}$in a Penning trap.

Figure 1 shows the change of the kinetic energy of the cyclotron motion as function of the applied frequency. The different figures correspond to dipole excitations with RFpulses following the time sequences shown in fig. 2. Figure 1a shows the energy absorption profile for a single RF-pulse of length $T_{r f}=920 \mathrm{~ms}$. The profile results directly from the Fourier transform of the RF-pulse

$$
E_{r} \propto\left[\frac{\sin \left(\pi \cdot \Delta \nu \cdot T_{r f}\right)}{\Delta \nu \cdot T_{r f}}\right]^{2}
$$

with $\Delta \nu$ being the detuning of the applied frequency from the cyclotron frequency. The width of the central peak is $\Delta \nu(F W H M) \simeq 0.9 / T_{r f}$. 
In the case that more than one excitation is applied to the trapped ions and that the phase of the oscillating field during the $n$-th excitation is fixed with respect to the first excitation, one expects to observe Ramsey fringes. In case of dipole excitation, the resulting line shape is again given by the Fourier transform of the excitation pattern. If the RF-field is switched on for a time $T_{r f}$ and the $n$ RF-pulses are repeated with a period $T$ one obtains for the radial energy the expression

$$
E_{r} \propto\left[\begin{array}{cc}
\sin \left(\pi \cdot \Delta \nu \cdot T_{r f}\right) & \sin (n \cdot \pi \cdot \Delta \nu \cdot T) \\
\Delta \nu \cdot T_{r f} & \sin (\pi \cdot \Delta \nu \cdot T)
\end{array}\right]^{2} .
$$

Figure $1 \mathrm{~b}, \mathrm{c}$ and $\mathrm{d}$ show the energy absorption profiles for $n=2,3,4, T_{r f}=130 \mathrm{~ms}$ and $T \cdot(n-1)=800 \mathrm{~ms}$. The narrowest width of the central peak is obtained for two excitations with $\Delta \nu(F W H M) \simeq 0.6 / T[1]$.

The situation is more complicated in case of quadrupole excitation: The energy absorption profiles do not simply result from the Fourier transform of the RF-pulses due to the nonlinear behaviour of the driven ion motion. An analytical expression has been obtained for a single excitation [11], the $n$-fold excitation is presently investigated.

\section{EXPERIMENTAL SET-UP}

The experimental set-up has been described in a number of publications [12,13,14]. Only the features needed in the context of this paper will therefore be reported in more detail. The Penning trap mass spectrometer is set up at the on-line facility ISOLDE at CERN which produces radioactive isotopes of a large number of elements. The aim of the experiment is to investigate the mass of these isotopes with highest accuracy. The set-up consist of two main parts; an ion cooler/buncher [15] connected to an ISOLDE beamline as well as to an ion source and the high-precision Penning trap for the mass determination. This trap is placed in the homogeneous part of the field of a superconducting 6-Tesla magnet. In the experiment discussed here the ion cooler/buncher delivers stable Cs ions from the test source as ion bunches to the high precision trap. Here the ions are captured in flight [12], trapped and subsequently excited. The ring electrode is split into four segments which allow the creation of electric dipole as well as quadrupole fields. The output of a frequency generator is switched according to the excitation patterns shown in fig. 2 and applied to the appropriate ring segments. After the excitation of their motion the ions are gently ejected out of the trap and drift through the inhomogeneous part of the magnetic field to an ion detector. In this field the ions' radial energy is converted into axial energy. In resonance this results in a reduction of the time of flight from the trap to the detector. 


\section{RESULTS}

Figure 3 shows cyclotron resonances obtained for ${ }^{133} \mathrm{Cs}$ ions. The measurements were performed following the time sequences for the RF-pulses shown in fig. 2. The upper part shows a $\nu_{+}$-resonance for a single RF-pulse, the lower figures the Ramsey fringes obtained with 2, 3 and 4 excitations. The solid curves are the result of a fit of the theoretical line shape to the resonances. The fitting function was obtained from the energy absorption profile (fig. 1 respectively eq. (1) and (2)) and a calculation of its nonlinear conversion into time of flight. Additionally the curves were folded by a Gaussian in order to account for the incomplete modulation of the fringes most likely due to the residual gas pressure. As can be seen, there is very good agreement between data and fit with the only adjusted parameters being the center frequency, the width of the Gaussian, the gained radial energy and four parameters for its conversion into time of flight. The fit error for the center frequency decreases steadily with the number of peaks in a resonance and is minimal in the case of the excitation with two RF-pulses (fig. 3b).

Finally figure 4 shows a resonance curve obtained by a quadrupole excitation of the ion motion at $\nu_{+}+\nu_{-}$. The excitation was performed with two RF-pulses according to the sequence shown in fig. $2 \mathrm{~b}$. For small amplitudes of the driving field the observed resonance approaches the shape of the Ramsey fringes obtained with dipole excitation. Therefore a good fit could be achieved with the same fitting function as used in fig. $3 \mathrm{~b}$.

\section{SUMMARY AND DISCUSSION}

The experiment performed here shows the direct observation of Ramsey fringes obtained from the motion of $\mathrm{Cs}$ ions in a Penning trap. The resonances are in perfect agreement with the theoretical prediction.

The aim of the mass experiment at ISOLDE is to measure accurately the mass of unstable isotopes during limited beam time. The Ramsey technique allows a reduction of the number of detected ions required for the determination of the cyclotron frequency with a certain statistical error. For an equal number of ions, the precision of the frequency determination increases with the number of the peaks in the Ramsey fringes. As in all mass experiments based on a Penning trap one limitation in the obtainable accuracy is frequency shifts caused by electric and magnetic field imperfections [9,10]. Other sources of frequency shifts are the image charge induced by the stored particle in the trap electrodes [16] and the effect of ion-ion interaction [11]. 
Ramsey fringes by dipole excitation of the cyclotron motion can be used as a tool in examining these effects. One possibility is the mapping of the electric and magnetic field in the plane perpendicular to the symmetry axis of the trap. The ions can be driven into a cyclotron motion of particular radius, stay in this orbit for a certain time while experiencing the present fields and can then be recentered with a RF-pulse phase shifted by $180^{\circ}$. The center of the Ramsey fringes as function of the amplitude of the driving RF-field shows then directly the radial dependence of $\nu_{+}$. For a perfect trap, $\nu_{+}$should be constant. The same scheme can be applied for the investigation of the effects of image charge, ion-atom, or ion-ion interaction. The latter has been found to cause frequency shifts if the ions differ in mass [11]. Again the influence on the eigenfrequency $\nu_{+}$can be directly investigated. 


\section{References:}

1) N. F. Ramsey, Phys. Rev., 76 (1949) 996

2) N. F. Ramsey, Rev. Mod. Phys., 62 (1990) 541

3) J. M. Pendlebury et al., Phys. Lett. 136 B (1984) 327

4) J. J. Bollinger et al., Phys. Rev. Lett. 63 (1989) 1031

5) L. G. Smith, Proc. of "3rd Int. Conf. on At. Masses" (1967), ed. R.C. Barber, Univ. of Manitoba Press, p.811

6) C. Thibault et al., Nucl. Phys. B (Proc. Suppl.) 8 (1989) 454

7) A. Cornell et al., Phys. Rev. Lett. 63 (1989) 1674

8) H. Stolzenberg et al., Phys. Rev. Lett. 65 (1990) 3104

9) L.S. Brown et al., Rev. Mod. Phys. 58 (1986) 233

10) G. Bollen et al., J. Appl. Phys. 68 (1990) 4355

11) M. König, diploma work, Mainz, and to be published

12) H. Schnatz et al., Nucl. Instr. and Meth. A251 (1986) 17

13) S. Becker et al., Int. J. Mass Spec. Ion Proc. 99 (1990) 53

14) H.-J. Kluge et G. Bollen, Nucl. Instr. and Meth., this volume ?????

15) G. Savard et al, Phys. Lett., in print

16) R. S. van Dyck, Phys. Rev. A40 (1989) 6308 


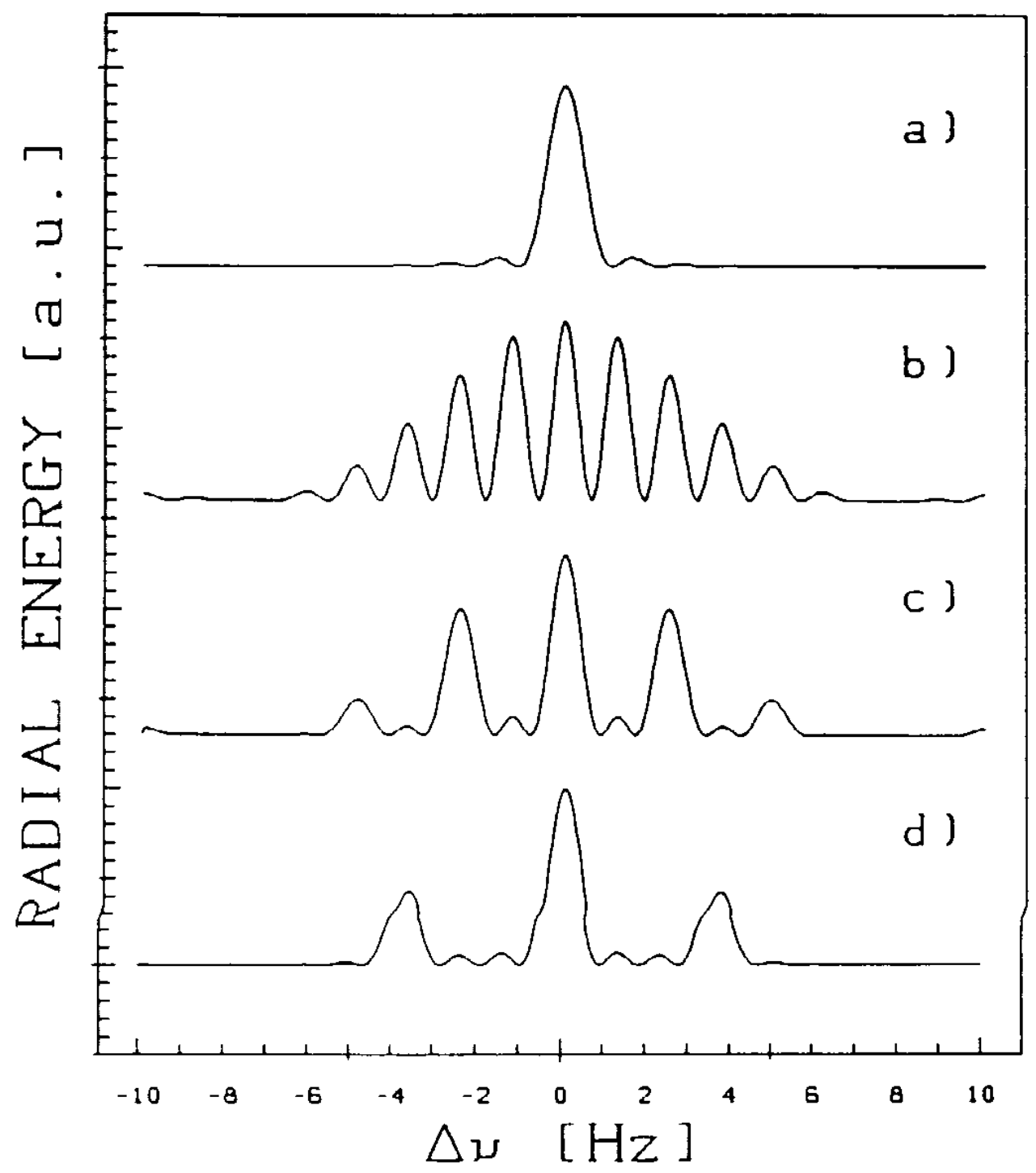

Fig. 1:

Energy absorption profiles in the case of dipole excitation of the cyclotron motion in a Penning trap. The profiles have been calculated by eq. (1) and (2) for the time sequences shown in fig. 2. 


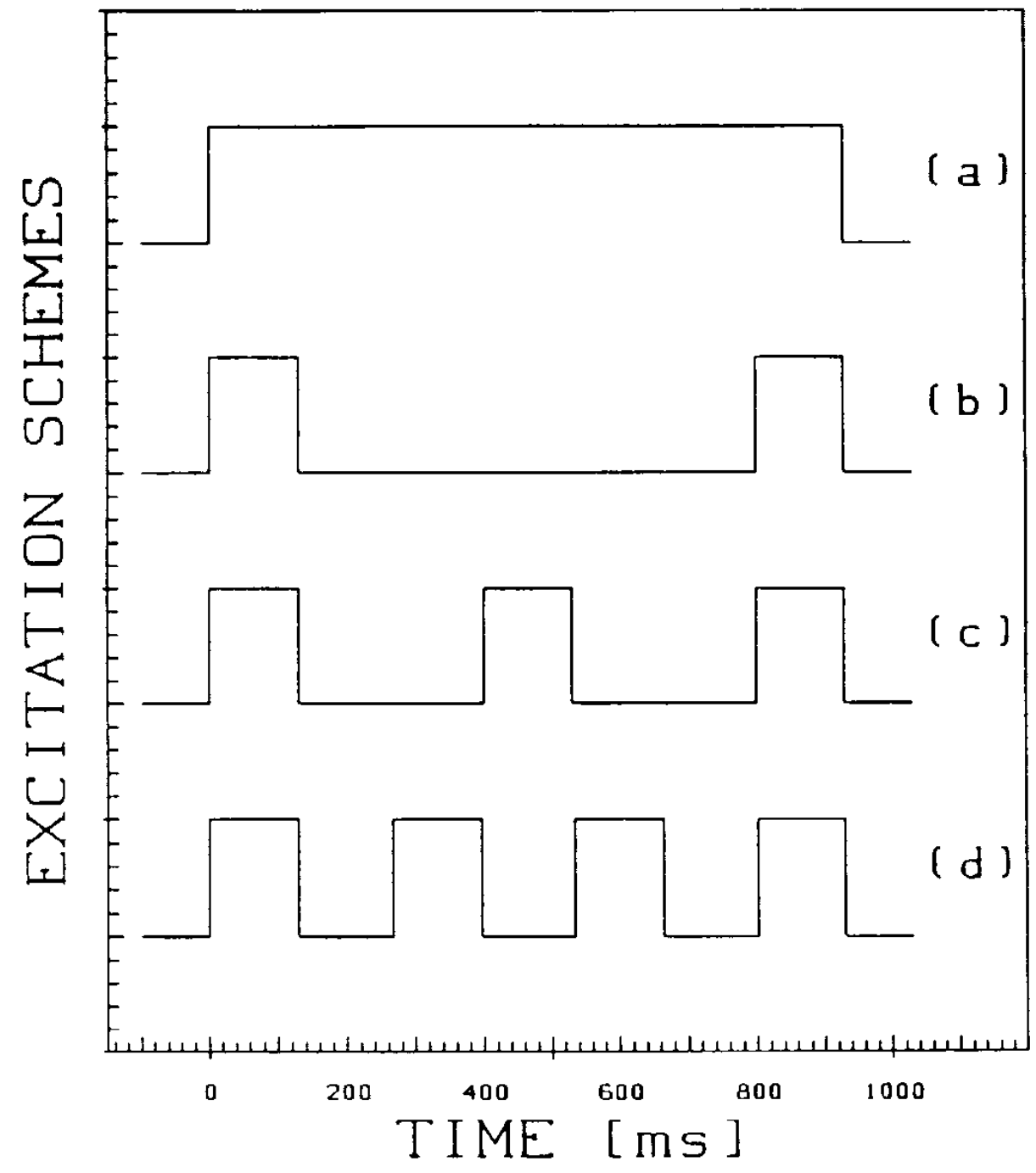

Fig. 2:

Time sequences of the RF-pulses for the excitation of the cyclotron motion with a) $n=1, T_{r f}=930 \mathrm{~ms}$, b) $\left.n=2, T_{r f}=130 \mathrm{~ms}, T=800 \mathrm{~ms}, \mathrm{c}\right) \mathrm{n}=3, T_{r f}=$ $130 \mathrm{~ms}, T=400 \mathrm{~ms}$, and d) $n=4, T_{r f}=130 \mathrm{~ms}, T=267 \mathrm{~ms}$. These sequences are underlying the energy profiles in fig. 1 and were used for the measurements. 


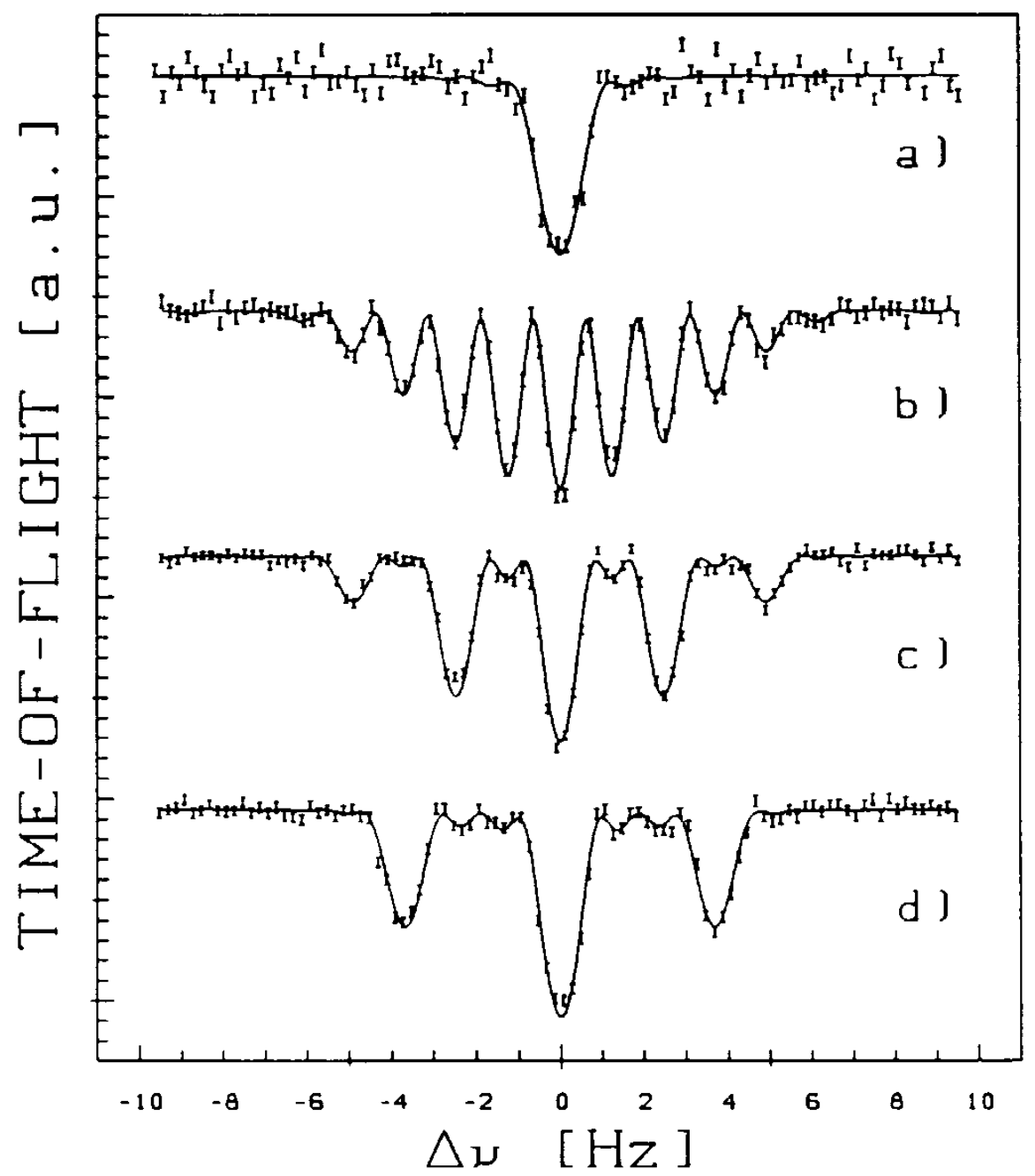

Fig. 3:

Cyclotron resonances of ${ }^{133} \mathrm{Cs}$ ions as obtained from the mean time of flight of ions from the trap to the detector as function of the detuning $\Delta \nu$ of the applied frequency with respect to the ions cyclotron frequency $\nu_{c}=683868 \mathrm{~Hz}$. The resonances were obtained by applying the excitation sequences shown in fig. 2 . The solid line correponds to a fit of the theoretical line shape to the data. 


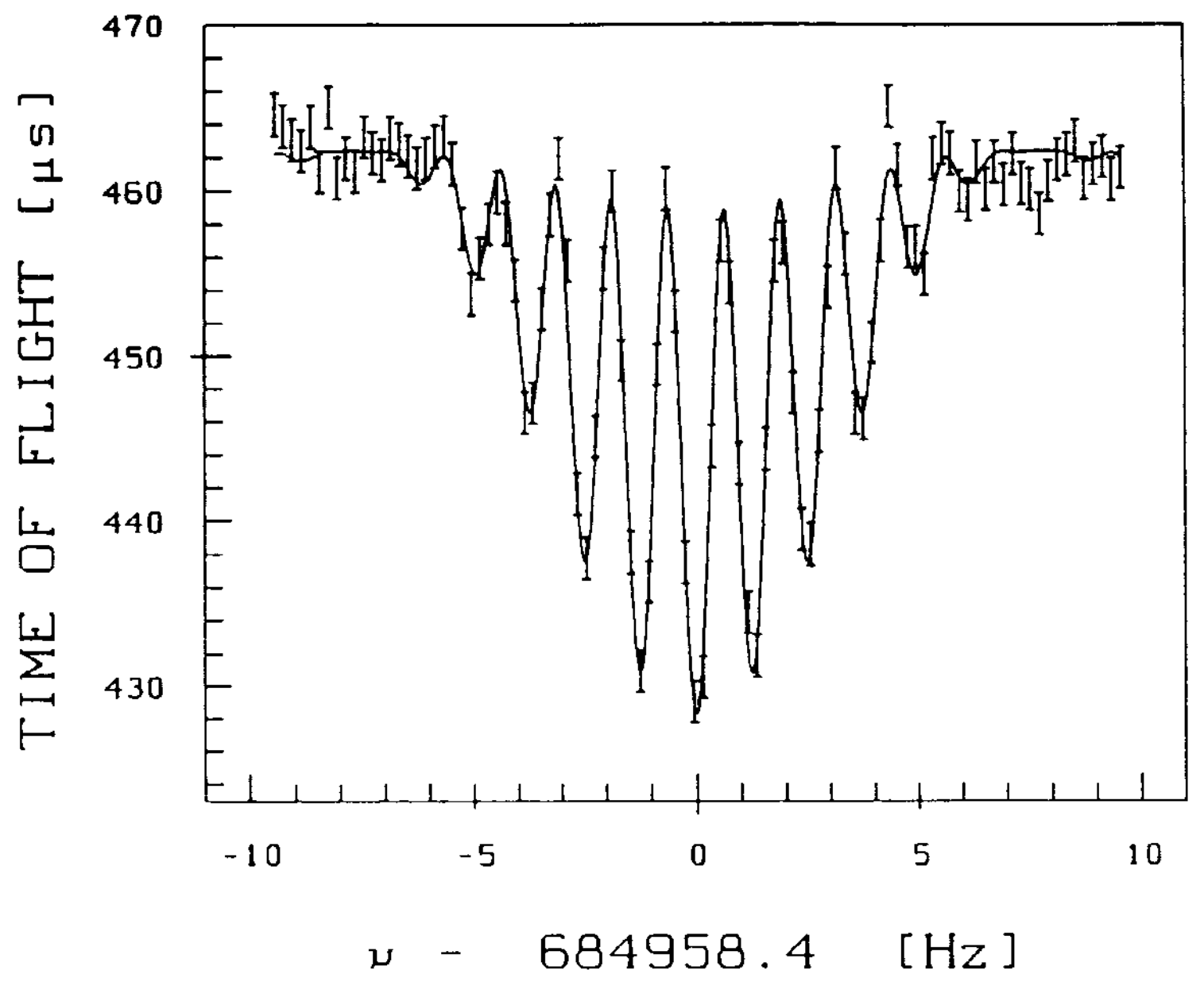

Fig. 4:

Cyclotron resonance at $\nu_{+}+\nu_{-}$obtained with quadrupole excitation. Excitation scheme b) from fig. 2 was used. The solid line corresponds to a fit with the sane fitting function as used in fig. $3 \mathrm{~b}$. 